\title{
The Study and Applications of Sparse Methods Technology in Yunnan Mountain Substation
}

\author{
Hongliang Wang1, Min $\mathrm{Cao}^{2}$, Xianfu Chen², Shilin $\mathrm{Li}^{2}$, Shaoquan Zhang², Xin Shen² \\ ${ }^{1}$ Postdoctoral Research Workstation of YunNan Power Grid Corporation, Kunming, China \\ ${ }^{2}$ Yunnan Electric Power Test \& Research Institute Group Co., Ltd., Electric Power Research Institute, Kunming, \\ China \\ Email: Hongliang.whl@gmail.com
}

Received January 2014

\begin{abstract}
As one of the divisions in China Southern Power Grid, Yunnan Power Grid Corporation has conducted research and demonstration projects on multiple smart grid technologies to improve the power system reliability, save operation cost and enhance measurement accuracy. In this paper, we will introduce The Study of Yunnan Mountain Substation Data Aggregation Technology based on Sparse Methods. Most substations are built in the mountain, the complex geological conditions and poor natural conditions put forward higher requirements on the substation running and real-time comprehensive monitoring of substation system. Processing and polymerization research of large amounts of the monitoring data and information is studied in this article. This paper introduces the sparse methods and then explains the thinning algorithm, especially new algorithm is proposed. Finally, the substation sparse method architecture is put forward and the simulation experiment was carried out to prove the feasibility and effectiveness of the proposed method.
\end{abstract}

\section{Keywords}

Sparse Methods; Mountain Substation; Geological Monitoring; Wireless Sensor Networks

\section{Introduction}

The 84\% mountain of Yunnan, topography, landform, geology, hydrology, climate and other complex conditions, determine the geological disease multiple and the necessity, urgency of monitoring in the process of construction and operation of Yunnan Mountain substation. These mountain substations inevitably slope. The stability condition of substations and transmission lines safe operations have a significant influence, and the monitoring of Yunnan substation geology has put a higher demand.

WSN technology is capable of acquiring and processing information reform on many occasions, widely used in environmental monitoring, resource monitoring, disaster pollution monitoring, public security and defense, intelligent transportation and other fields, can be arranged in harsh, demanding, complex environment for moni- 
toring and detection in various condition. The WSN network information processing technology has made great progress, but there are some issues have not yet been fully resolved, such as: shorten the delay, delay distribution reasonable, appropriate data distribution mechanism, and ensure the robustness, low data transmission based on QoS.

The temporal and spatial sampling network, sensor node energy and bandwidth are very limited, but require periodic sensing and transmitting massive data, and often faced with data loss and nodes energy less these two problems. In order to reduce the consumption of resources (usually refers to energy) and the choice of intermediate nodes for data processing, to prolong the lifetime of network technology, domestic and foreign experts and scholars have made a lot of research. Tiny D uses tree structure distribution request and collect data to Sink node [1]. Some scholars put forward the outline diffusion algorithm, by designing reach sequence and the polymerization mechanism ODI (order and duplicate insensitive) of and repeat information insensitive to reduce the message routing data aggregation. Cheng Ziyao, Liu Yun uses a mobile sink node and a static sink node coexistence mode, presents an efficient data collection method of HS BDM (Hybrid Sinks Based Dissemination Management), the simulation shows that the mobile sink node data collection in saving energy is obviously superior to the traditional network, at the same time, prolong the survival time of the network can be obtained the high success rate of data transmission and shorter delay of data transmission [2].

But the Yunnan special, complex geographical environment makes the substation construction not centralized, decentralized, in wireless sensor network deployment process of substation geological monitoring should cover a vast area for collecting all kinds of information, and constitute a less dense network, as sparse wireless transmission network. In actual geological monitoring system diagnosis, geological disasters often manifested as a long and gradual process, only a single feature and monitoring method is unable to complete the monitoring task. Sparse methods can be more accurate and deeply cognize the geological monitoring information of substation, to meet the practical needs of the substation geological monitoring, more and more people are paying attention to this.

\section{Sparse Methods Theory}

Study of sparse method can be traced back to the last century ninety's, the sparse signal representation to the large volume of data transmission and storage, and in recent years the rise of compressed sensing theory to make the sparse representation has been paid more and more attention. The sparse representation theory that the signal can be sampled at less than Nyquist sampling frequency, and can accurately reconstruct the original signal, when it was proposed, it has been widely used in the signal processing field [3].

So-called signal sparse representation, in the transform domain, using the transform basis as little as possible to represent the original signal, the sparse representation is the process of signal sparse decomposition. Usually consider the linear combination, namely $y=A x$ form. The $y$ is target signal vector, $A$ is transform based, $x$ is $y$ linear in this group based on the expansion coefficient vector. If signal $y$ based on A the coefficient vector $x$ in the non zero term rarely, that there are many terms are all zero, then in accordance with the definition of sparsity $x$ is sparse, establishing the model $y=A x$ is signal $y$ sparse representation process. This signal can be expressed as [4]:

$$
y=\sum_{i=1}^{N} A_{i} x_{i}
$$

The representation of a signal is essentially the signal in a known set of functions were decomposed, and then the expression of the original signals in transform domain. In the description of a signal, if the parameters are less, the signal is sparse; the sparseness of some signal can be directly displayed, but some signal need put through some transformation that characteristics of the sparse can be expressed. Therefore we need to find a suitable signal sparse transform, to make the sparse expression [5].

Data aggregation of wireless sensor networks is a technology that in the collection and dissemination of information in a multi-hop networks, to reduce the resource consumption (usually refers to energy) and select data processing in the middle node, and in order to prolong the network life technology. The network polymerization technology consists by suitable network protocols, efficient aggregation function and effective data representation of three basic parts. The traditional network routing always transfer data along the shortest path, and in WSN, we minimize energy consumption is the purpose of data aggregation. 


\section{Sparse Algorithm Research}

\subsection{Curvelet Discrete Cosine Transforms}

In view of the core parts of the first generation of curvelet transform are the subband decomposition and ridgelet transform, and its biggest flaw is to realize the more complex, the whole process will produce tremendous data redundancy. Mentioned in this article, therefore, the curvelet transform refers to the second generation of curvelet transform. Through the signal and inner product in the form of a wave function can realize the signal sparse:

$$
c(j, l, k)=\left\langle f, \varphi_{j, l, k}\right\rangle
$$

$\varphi_{j, l, k}$ is curvelet function, $j, k, l$ is the scale, direction and position parameter respectively. Inverse discrete curvelet transform formula is [6]:

$$
f^{\prime}=\sum c(\mathrm{j}, \mathrm{l}, \mathrm{k}) \cdot \varphi_{j, l, k}
$$

$c(\mathrm{j}, \mathrm{l}, \mathrm{k})$ is curvelet coefficient processed in the curvelet domain, $\varphi_{j, l, k}^{\prime}$ is the inverse wave function, $f^{\prime}$ is the result of inverse discrete curvelet transform.

Curvelet transform implemented in frequency domain, window function $U$ of frequency domain is used to represent $\varphi$ in the frequency domain. To define a pair of window function: the radial window function $W_{r}, r \in(1 / 2,2)$ and angle window function $V_{(\mathrm{t})}, t \in[-1,1]$. They all meet the permissible conditions:

$$
\begin{aligned}
& \sum_{j=-\infty}^{+\infty} W^{2}\left(2^{\prime} \mathrm{r}\right)=1, r \in(3 / 4,3 / 2) \\
& \sum_{j=-\infty}^{+\infty} V^{2}(t-l)=1, \mathrm{t} \in(-1 / 2,1 / 2)
\end{aligned}
$$

For each $j \geq j_{0}$, frequency window $U_{j}$ in the frequency domain defined is as follows:

$$
\mathrm{U}_{j}(\mathrm{r}, \theta)=2^{-3 j / 4} W\left(2^{-j} \mathrm{r}\right) \mathrm{V}\left(\frac{2^{[j / 2]} \theta}{2 \pi}\right)
$$

Then signal processed by discrete cosine transform. The discrete cosine transform is a kind of the definition of transformation of the real signal, after the transformation in the frequency domain is also a real signal, but because of the discrete cosine transform is equivalent to discrete fourier transform about its twice length, the discrete fourier transform was conducted for a real even functions, so compared with discrete fourier transform can reduce more than half of the data redundancy [7].

Discrete cosine transform one dimensional computation formula is as follows:

$$
X(\mathrm{k})=\sum_{n=0}^{N-1}\left(\mathrm{c} \sqrt{\frac{2}{N}}\right) x(\mathrm{n}) \cos \frac{k(2 \mathrm{n}+1) \pi}{2 N},(\mathrm{n}=0,1 \cdots, \mathrm{N}-1)
$$

here:

$$
c=\left\{\begin{array}{c}
1 / \sqrt{2}, n=0 \\
1, n \neq 0
\end{array}\right.
$$

Formula (6) for signal processing and image processing, due to the discrete cosine transform has the characteristics of a strong "the energy concentration" and when the signal is close to statistical properties of the Markov process, discrete cosine transform to correlation is close to $\mathrm{K}$ - L transform, it is optimal to correlation performance.

\subsection{Substation Sparse Method Architecture}

Sparse method information aggregation structure of substation is shown in Figure 1 [8].

\section{Testing and Simulation}

In WSN, usually a sparse node layout detect small probability event. In this case, each node should be based on 


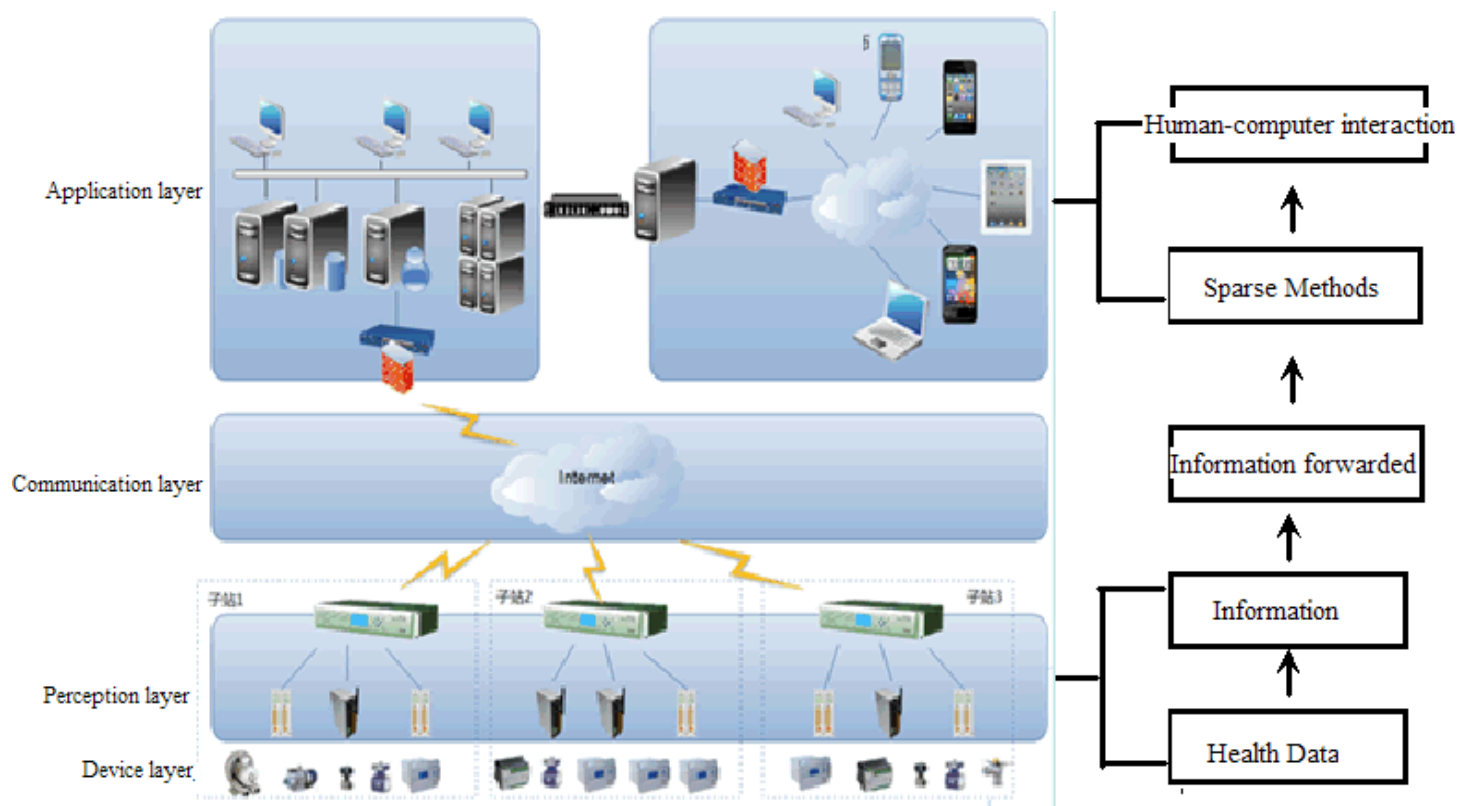

Figure 1. Sparse method information aggregation framework of substation.

their perception of the data into the data aggregation. Such as ChuXiong mountain waist substation exploits optical fiber Bragg grating strain sensor on-line monitoring geological parameters temperature, stress, and fracture and shifting and high efficiency for these large amounts of data aggregation.

Assume that in the case of sensor nodes meet the connectivity and coverage, to a certain density uniformly distributed in a rule area, the boundary of the area is known, and nodes distributed on the boundary connected to external high-speed network. Regional nodes according to their own monitoring data to determine whether there are hot nodes. Algorithm's goal is to count the number of network and internal nodes and give a more general polymerization method based on the statistical characteristics of hot nodes. These statistical properties may include the node number, the location of node or secondary measured values used to transmit more monitoring information. In order to get the sensitive features distance, each participating node along the double rulings trajectory sending probe packets in probing protocol.

A mountain substation in Yunnan retaining wall distributed sensor layout diagram is shown in Figure 2.

Node acquired the equipment status information from all kinds of instruments and sensors are generally large, difficult to understand the data. In order to adapt to the need of data aggregation level processing, acquisition node should complete classification and pretreatment of raw data, simple calculation and judgment of the original data, to collect datum contain useful information for facilitating node calculation.

A mountain substation in Yunnan retaining wall measured stress on sparse distributed sensor method is shown in Figure 3.

Blue line-stress the original waveform, the red line-sparse transformation result, appeared two smaller mutations in the 110 days, 130 days or so, appeared continuous abnormalities during the period of 190 days to 210 days. The geological exploration proves strain response happens in the 195th day. Figure 3 shows that the method of sparse transformation accurately calibrated the abnormal phenomenon began in the 195th day, ended in the 210th day, and filtered other small mutations of the original waveform. According to the initial conditions of the data screening "monitoring for 5 days, each component rate $<10 \%$ in the adjacent two days are seen as normal”, the results of the sparse method transform conform to the judgment condition.

\section{Conclusion}

This paper introduces the sparse method theory, and then explains the thinning algorithm, especially new algorithm. In the end, the substation sparse method architecture is put forward and a simulation experiment was carried out. Through these experiments, the proposed method is to be proved feasibility and effectively. 


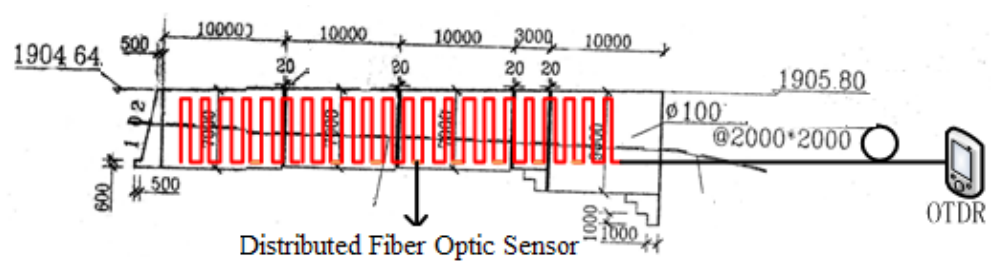

Figure 2. A mountain substation of Yunnan retaining wall distributed sensor layout diagram.

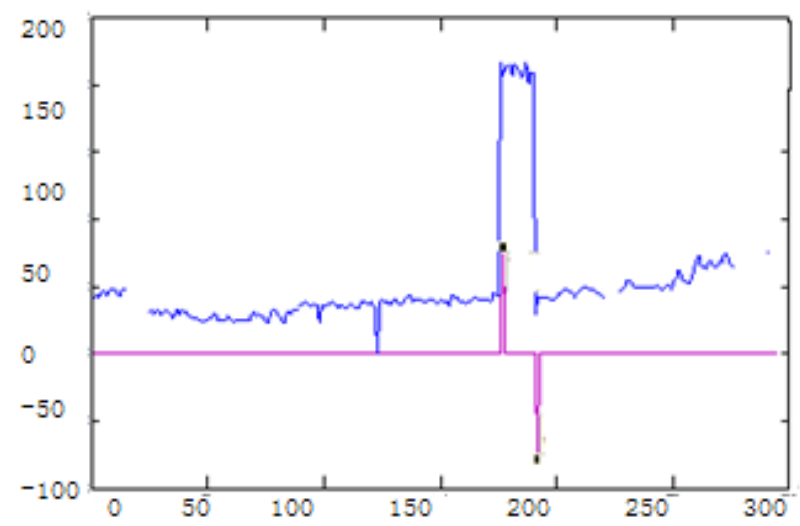

Figure 3. A mountain of Yunnan substation retaining wall distributed sensor layout diagram (Red Line stands for Sparse Methods, blue line stands for stress test).

\section{Acknowledgements}

Paper is supported by the China Postdoctoral Science Foundation (2013M540721) and National High-Tech Research and Development Program (863) of China (2011AA05A120).

\section{References}

[1] Madden, S.R., Franklin, M.T., Hellerstein, J.M. and Hong, W. (2002) TAG: A Tiny Aggregation Service for Ad-Hoc Sensor Networks. Proceeding of the Symposium on Operating Systems Design and Implementation, OSDI.

[2] Wang, H.L., Dong, H.B. and Jiang, G.S. (2012) Design of EM-MWD Signal Detection System Based on Correlation and Adaptive Filter. Chinese Journal of Scientific Instrument, 5, 1013-1018.

[3] Wang, H.L., Dong, H.B., et al. (2013) The Study of Cased-Hole Transient Electromagnetic Pulse Acquisition Unit. Oil Geophysical Prospecting, 48, 804-808.

[4] Cheng, Z.Y. and Liu, Y. (2009) An Efficient Data Collection Method by Mobile Sink Node in Wireless Sensor Networks. Journal of Beijing Jiaotong University.

[5] Wang, H.L., Dong, H.B., et al. (2012) The Study of FID Signal Extraction Technology of Proton Magnetometer Based on SVD. International Journal of Digital Content Technology and its Applications, 10, 576-584.

[6] Wang, H.L., Dong, H.B., He, L.H., Shi, Y.L. and Zhang, Y. (2010) Design and Simulation of LQR Controller with the Linear Inverted Pendulum. International Conference on Electrical and Control Engineering, 6, 699-702.

[7] Wang, H.L. and Dong, H.B. (2012) Study of the Distributed Ground-Tunnel Pressure Measurement and Control Method. Applied Mechanics and Materials, 48-51.

[8] Nath, S., Gibbons, P.B., Seshan, S. and Anderson, Z.R. (2004) Synopsis Diffusion for Robust Aggregation in Sensor Networks. SenSys'04: Proceedings of the 2nd International Conference on Embedded Networked Sensor Systems, 250262. http://dx.doi.org/10.1145/1031495.1031525 